\title{
Amas de blocs cyclopéens sur l'île de Banneg (Archipel de Molène - Finistère). Etude morpho-sédimentaire et dynamique de mise en place
}

\author{
Bernard Fichaut, Serge Suanez \\ Maîtres de Conférence à l'Université de Bretagne Occidentale \\ GEOMER - UMR 6554 LETG - CNRS - Institut Universitaire \\ Européen de la Mer, Place Nicolas Copernic, 29280 Plouzané. \\ bernard.fichaut@univ-brest.fr, serge.suanez@univ-brest.fr
}

\begin{abstract}
Résumé :
L'île de Banneg, située à la limite nord ouest du plateau de Molène, reçoit de plein fouet l'attaque des houles d'ouest. A l'occasion de fortes tempêtes, des dalles de roches sont arrachées aux falaises et projetées jusqu'à 11 mètres d'altitude à l'intérieur de l'île où elles forment des amas de blocs cyclopéens dont le volume total avoisine $900 \mathrm{~m}^{3}$. Les tempêtes de l'hiver 1989-1990 ont totalement remanié ces amas et ont apporté de nouveaux dépôts. L'analyse des données marégraphiques et des houles enregistrées durant cette période, montre que des vagues atteignant $18 \mathrm{~m}$ de hauteur au large, associées à des niveaux de PM d'environ $4 \mathrm{~m}$, sont à l'origine de niveaux marins extrêmes proches de $14 \mathrm{~m}$ (IGN 69) entraînant une submersion partielle de l'île.
\end{abstract}

\begin{abstract}
:
Banneg island, located on the north west limit of Molène submerged platform, receives the full impact of western swells. During strong storms, boulders are torn away from the cliffs and thrown up to 11 meters high ontop the island were they form boulder ramparts totalizing $900 \mathrm{~m}^{3}$. The storms of winter 1989-1990 have completely modified the topography of the existing ramparts and moved inshore new boulders. The analysis of forcings (wave and tide) recorded during this period, shows that deep water waves reaching $18 \mathrm{~m}$ high, combined to high tide level of about $4 \mathrm{~m}$, induce extreme water levels close to $14 \mathrm{~m}$ involving partial submersion of the island.
\end{abstract}

Mots clefs : barrages de blocs cyclopéens, runup, submersion de tempête.

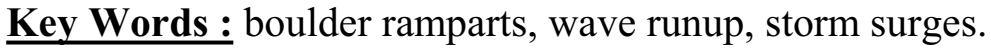

\section{$\underline{\text { 1. Introduction }}$}

L'île de Banneg, partie intégrante de la Réserve Naturelle d'Iroise, est située dans l'archipel de Molène, au large de la côte nord ouest du département du Finistère, en Bretagne (fig. 1). Les 19 îles et îlots de l'archipel dont la superficie totale est de 195 ha ne représentent que la partie émergée d'un vaste plateau sousmarin qui s'étend sur environ 15000 ha au dessus de l'isobathe $10 \mathrm{~m}$. Banneg se 
distingue des autres îles, relativement abritées, par sa position excentrée au nord ouest, immédiatement en bordure du profond chenal du Fromveur. Les isobathes 20 et 50 mètres sont respectivement à $250 \mathrm{~m}$ et $2200 \mathrm{~m}$ de la côte occidentale de l'île qui reçoit de plein fouet les houles dominantes de ce secteur. Lors de très fortes tempêtes, les déferlements sont suffisamment puissants pour arracher à la falaise et projeter à l'intérieur de l'île des blocs formant des amas, voire des barrages (fig. 2). Ces derniers sont appelés blocs cyclopéens en référence aux murs cyclopéens de la ville fortifiée de Mycènes ${ }^{[1]}$. Nous présenterons tout d'abord la morphologie et la disposition de ces édifices, avant de nous intéresser aux processus hydrodynamiques responsables de leur mise en place.

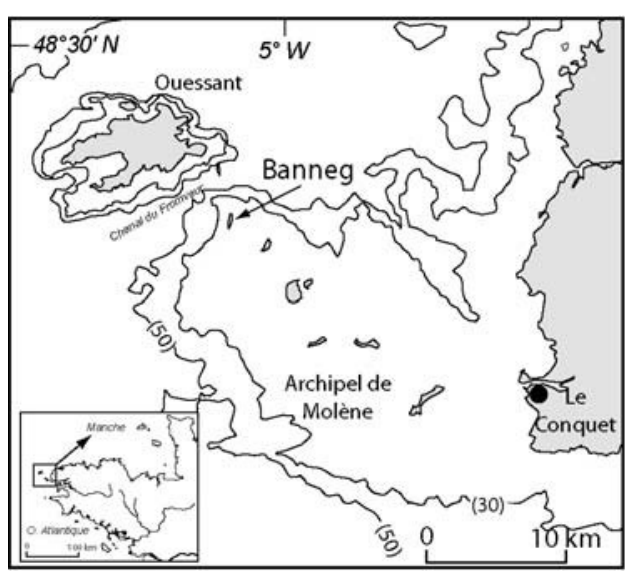

Figure 1: Carte de localisation

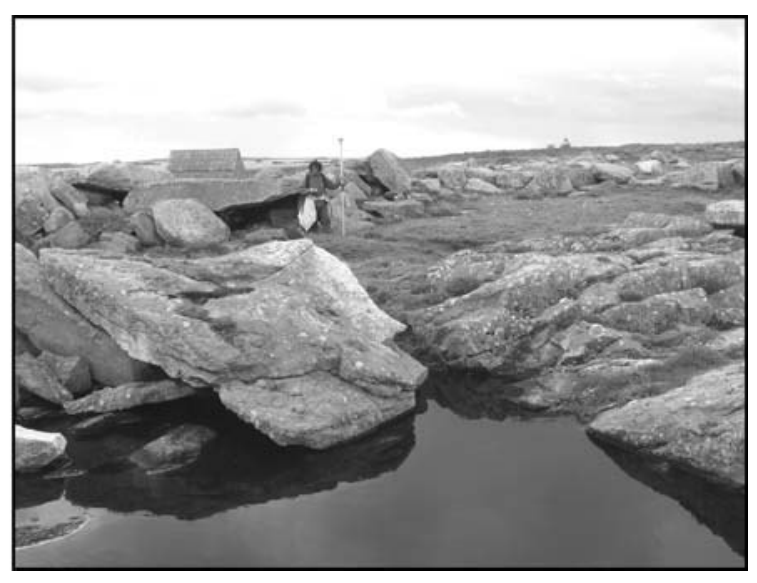

Figure 2: Barrage de blocs cyclopéens au centre de Banneg à 9 m d'altitude (cliché : B. Fichaut - 2005).

\section{Morphologie de Banneg}

A l'automne 2005 un relevé topographique au DGPS de l'île a été réalisé. Plus de 15000 points ont été levés sur 9 ha que représente sa superficie, permettant ainsi de restituer finement sa morphologie (fig. 3). Longue de 800 mètres et large de 300 mètres au maximum, Banneg s'étire selon un axe nord sud et présente une nette dissymétrie. La côte est, sous abri, est formée d'un estran essentiellement rocheux, localement recouvert de placages de galets et blocs ou de sable. Cette côte basse se raccorde à l'intérieur de l'île par un long versant peu pentu. La côte ouest est entaillée en falaises. Subverticales et atteignant 15 mètres de hauteur au niveau des pointes, elles sont moins élevées et ont un profil en marches d'escaliers dans les rentrants. Cette topographie résulte de la structure du granite en place, constitué de dalles subhorizontales individualisées par le réseau de diaclases. A l'emplacement des failles, d'orientation NW-SE, de longs couloirs d'érosion ont été évidés. Le socle rocheux affleure presque partout dans la zone intertidale où les accumulations se limitent à d'énormes boules granitiques de plusieurs tonnes disséminées çà et là sous le niveau moyen des pleines mers. 


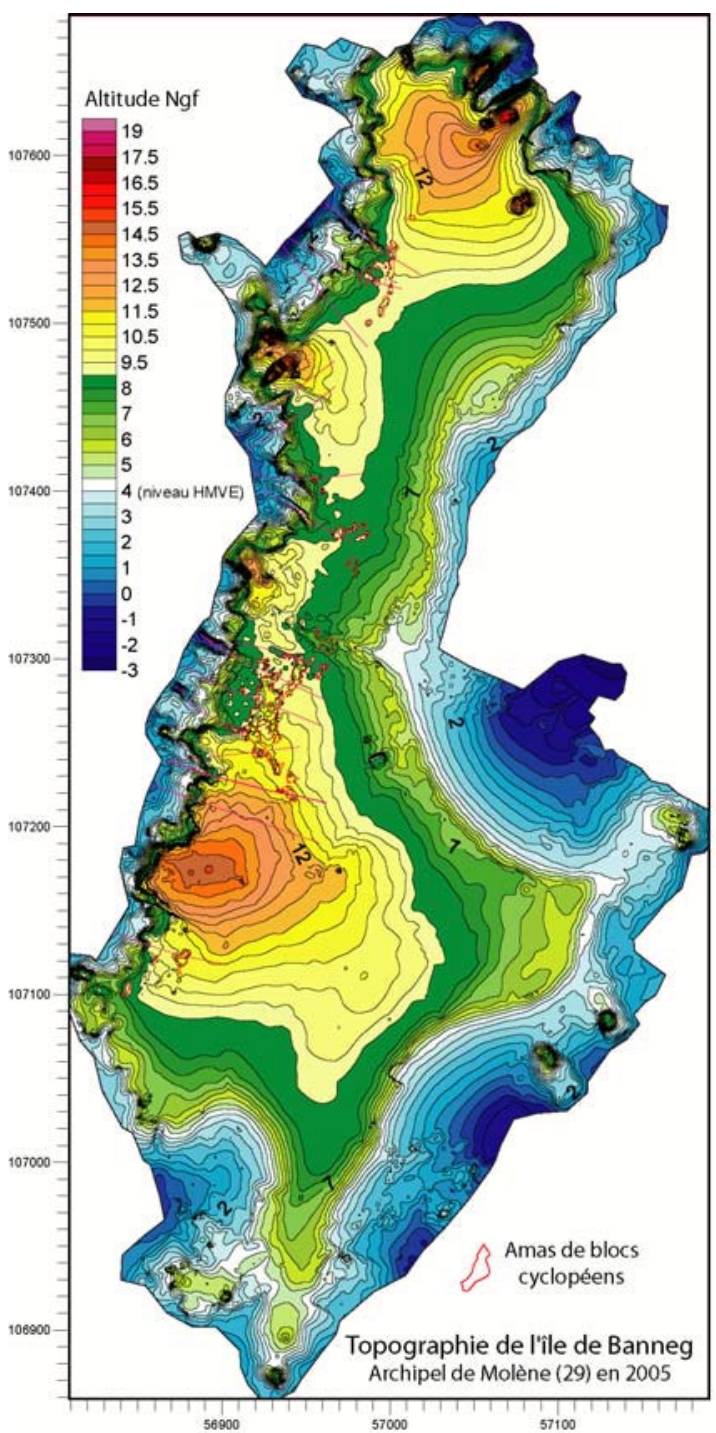

Figure 3: Topographie de l'île de Banneg restituée à partir d'un MNT levé au DGPS

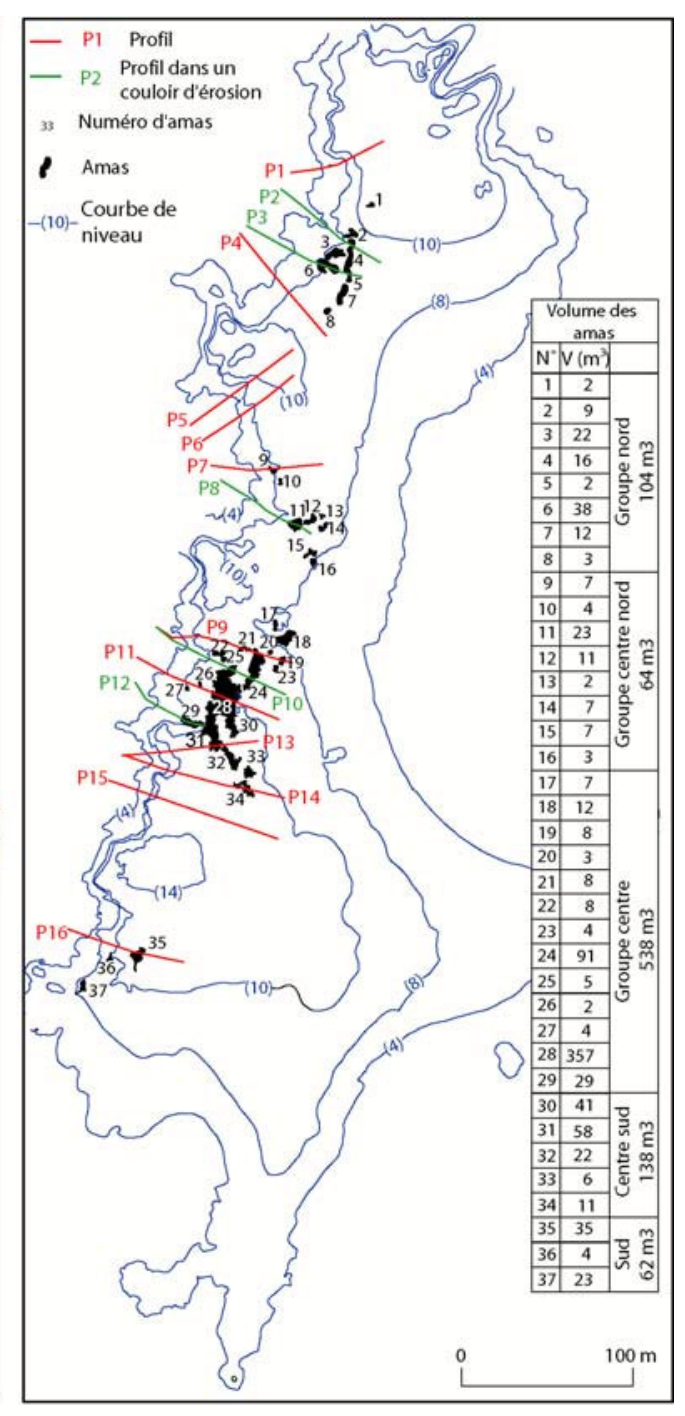

Figure 4 : Localisation, volumétrie des amas de blocs cyclopéens de l'île de Banneg

\section{$\underline{\text { 3. Les amas de blocs cyclopéens, localisation et caractéristiques }}$}

L'originalité de la morphologie de la côte ouest consiste en la présence d'amas de blocs cyclopéens situés sur la partie sommitale de l'île, à des altitudes comprises entre 7,5 et 11,2 m IGN 69 (fig. 3 et 4). Certains sont immédiatement en haut de falaise, les plus éloignés sont à 60 mètres de celle-ci. A quelques exceptions près, les blocs sont anguleux contrairement à ceux qu'on trouve dans la zone intertidale. Il s'agit donc pour l'essentiel de dalles arrachées à la falaise et projetées par les vagues vers l'intérieur de l'île. Leur poids unitaire variant de quelques centaines de kilos à 7,6 tonnes pour la plus grosse, déposée à 35 mètres $\mathrm{du}$ rebord de l'escarpement. Le volume total des amas est de $900 \mathrm{~m}^{3}$, vides interstitiels compris.

Ils sont répartis en 5 groupes reposant au droit des rentrants de la côte (fig. 4). Trois facteurs seulement s'opposent à leur présence. Ils sont absents $(i)$ en arrière 
de sommet de falaise d'altitude supérieure à 11 mètres (P5 et 15 fig. 5A), (ii) lorsque la côte fait face au nord, (iii) ou lorsqu'elle est abritée immédiatement en arrière des pointes $(\mathrm{P} 4$, fig. $5 \mathrm{~A})$.

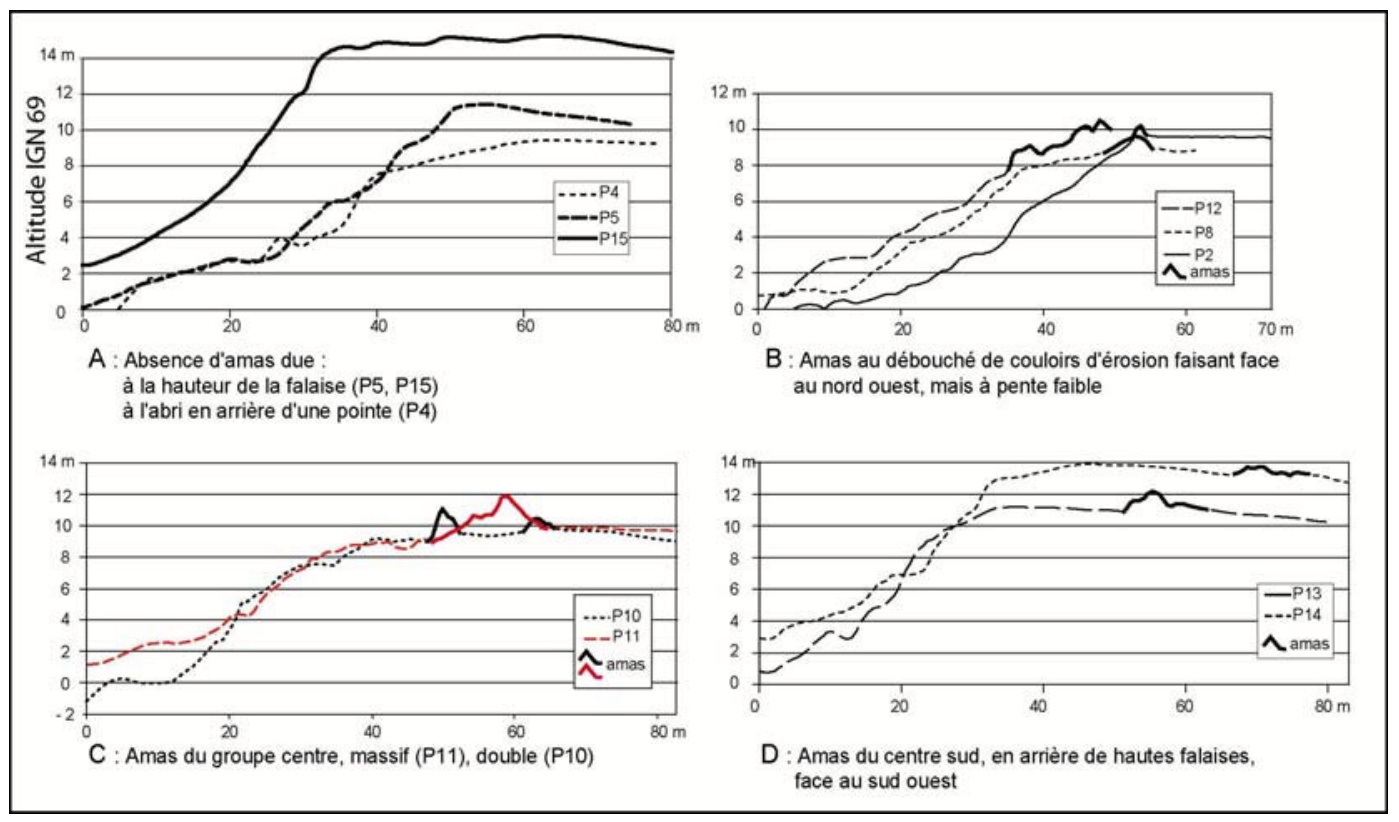

Figure 5: Profils représentatifs de la topographie de la côte ouest de Banneg et de la localisation des amas de blocs cyclopéens

Aucun autre paramètre ne semble limitant. En effet les amas font face à toutes les directions du fetch du quadrant sud ouest - nord ouest (tab. 1).

Face au nord ouest qui n'est pas dans l'axe du fetch maximum, ils sont souvent au débouché des couloirs d'érosion entaillés jusqu'en sommet de falaise, et dans lesquels la pente est faible, inférieure à 33\% (fig. 5B). Ces couloirs, qui canalisent les déferlements, fonctionnent comme des rampes ascensionnelles. Localement, comme dans l'axe des profils 8 et 10, les couloirs débouchent, au sommet de l'île, vers $8 \mathrm{~m}$ d'altitude dans des amorces de «vallons » qui ont été creusés par l'écoulement de l'eau de mer. En effet, lors de certaines tempêtes, l'eau des déferlements traverse l'île par ces passages pour s'écouler vers la côte occidentale ${ }^{[2]}$. Dans ce cas, les blocs sont entrainés par la lame d'eau et déposés sous forme d'amas isolés jusqu'au centre de l'île (amas 11 à 18, fig. 4).

Les accumulations les plus importantes sont au centre de l'île. Là, les édifices atteignent 2,5 mètres de hauteur et 20 mètres de large pour un volume de plusieurs centaines de mètres cubes (amas 24 et 28 fig 3, fig 5C). Localement, il y a même deux barrages parallèles qui montrent qu'à certaines occasions la masse et la puissance du jet de rive sont telles que les projections se font sur des distances plus importantes. L'importance des accumulations dans cette partie de l'île n'est pas uniquement liée à la faible hauteur de l'escarpement $(<9 \mathrm{~m})$, ce qui est aussi le cas plus au nord. Elle s'explique également par l'attaque directe des houles d'ouest et ouest sud-ouest alors que plus vers le nord, l'orientation générale du trait de côte NNE - SSW, et la présence de pointes entraînent une réfraction / diffraction des houles et diminuent la puissance du déferlement. 
Les amas du centre sud (fig. 4 et 5D) sont les seuls disposés face à l'ouest sudouest. La position de l'amas 34, à 33 mètres en retrait d'un bord de falaise atteignant 11 mètres d'altitude montre que les blocs ont franchi un dôme à $12 \mathrm{~m}$ avant de se déposer (tab. 1). Localement, les amas forment même un double barrage, le second se trouvant à 40 mètres de l'escarpement de la falaise. L'orientation face à l'ouest sud-ouest est donc une situation très favorable. L'absence d'édifices sur les autres portions de côte orientées de la même manière, découle de la trop grande hauteur des falaises sur la face sud des pointes.

La petitesse des édifices du groupe sud, malgré l'orientation et l'altitude propice au franchissement des vagues, s'explique par la présence de hauts fonds prolongeant vers le large la pointe la plus occidentale de l'île.

\begin{tabular}{|l|c|c|c|c|c|c|}
\hline $\mathrm{N}^{\circ}$ & Orientation & $\begin{array}{c}\text { Hauteur de la } \\
\text { rupture de pente } \\
\text { en haut de falaise }\end{array}$ & $\begin{array}{c}\text { Hauteur } \\
\text { de la base } \\
\text { des amas }\end{array}$ & $\begin{array}{c}\text { Distance } \\
\text { amas - haut } \\
\text { de falaise }\end{array}$ & $\begin{array}{c}\text { Pente } \\
\text { de la } \\
\text { falaise }\end{array}$ & $\begin{array}{c}\text { Dénivellée } \\
\text { de la } \\
\text { falaise }\end{array}$ \\
\hline P1 & WSW & 10 & - & - & $42 \%$ & 6 \\
\hline P2 & NW & 9,6 & 9,6 & 0 & $33 \%$ & 6,4 \\
\hline P3 & NW & 8 & 8 & 0 & $16 \%$ & 5,2 \\
\hline P4 & NW & 7,5 & - & - & $41 \%$ & 3,5 \\
\hline P5 & SW & 11,3 & - & - & $33,5 \%$ & 8,5 \\
\hline P6 & SW & 8,2 & - & - & $31 \%$ & 6,2 \\
\hline P7 & W & 7,6 & - & - & $82 \%$ & 5,4 \\
\hline P8 & NW & 7,8 & 8,8 & 12 & $27 \%$ & 6,9 \\
\hline P9 & WNW & 8 & $8,7+9+8,3$ & $25+34,5+50$ & $52 \%$ & 7,8 \\
\hline P10 & WNW & 9,1 & 9 et 9,7 & 8 & $32 \%$ & 9 \\
\hline P11 & WNW & 8,4 & 9 & 15,3 & $36 \%$ & 4 \\
\hline P12 & WNW & 7,5 & 7,5 & 0 & $23 \%$ & 7,5 \\
\hline P13 & WSW & 11,5 & 10,9 & 18 & $33 \%$ & 7 \\
\hline P14 & WNW & 10,9 & 11,2 & 33,5 & $42 \%$ & 5 \\
\hline P15 & WNW & 12,5 & - & - & $35 \%$ & 12 \\
\hline P16 & WNW & 9,8 & 10,5 & 15,5 & $73 \%$ & 4,6 \\
\hline
\end{tabular}

Tableau 1 : Caractéristiques de la localisation des amas de blocs sur la côte ouest de Banneg. Distances et hauteurs en mètres. Sur les profils P9 et P10 on trouve respectivement 3 et 2 amas sur le profil.

\section{Mise en place et dynamique des amas.}

La littérature relative à la mise en place d'amas de blocs cyclopéens tend à expliquer leur présence par le déferlement de vagues de tsunami ${ }^{[3][4]}$ ou d'ouragans tropicaux ${ }^{[5]}$. Dans certains cas, les auteurs arrivent à dater l'événement responsable de leur édification ${ }^{[5]}$, dans d'autres, ils n'aboutissent qu'à une estimation ${ }^{[4]}$. Toutefois, l'ancienneté des datations généralement avancées (plusieurs dizaines à centaines d'années) ne permet pas de caractériser précisément les conditions hydrodynamiques responsables de ces dépôts. Dans le cas de Banneg, les observations menées depuis ces dernières décennies dans le cadre de la réserve naturelle ont permis d'analyser et de dater précisément quelques épisodes morphogènes ${ }^{[6]}$. Ainsi, l'étude diachronique menée par photointerprétation montre l'importance des changements morphosédimentaires sur plusieurs secteurs de l'île entre 1979 et 2005 (fig. 6A et 6B). Ces derniers sont 
attribués à l'action des tempêtes de l'hiver 1989-1990 dont les caractéristiques hydrodynamiques ont été analysées.

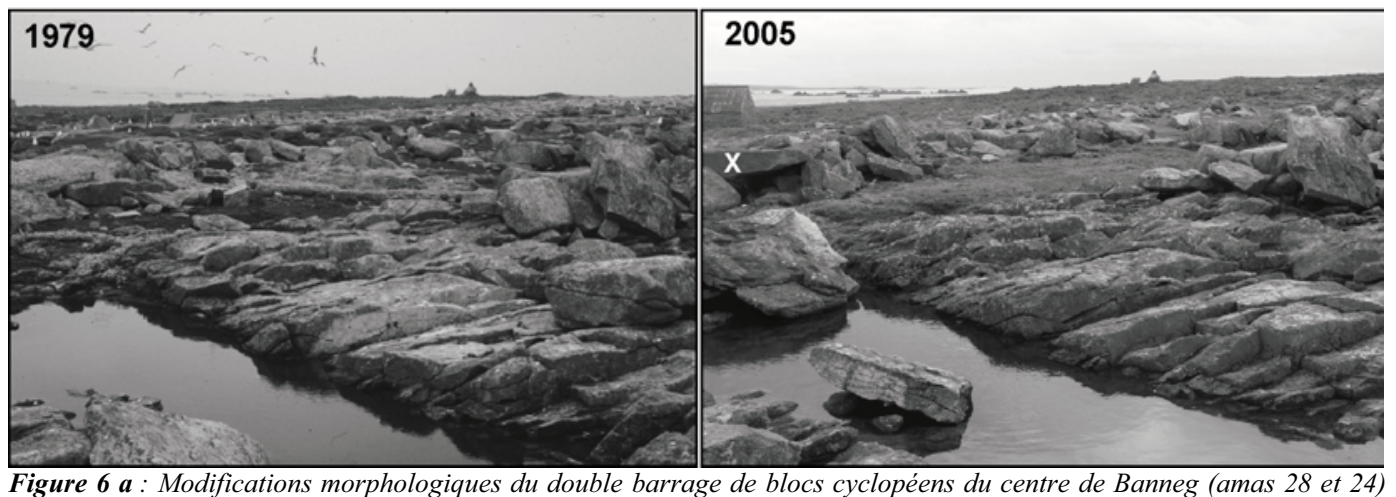
intervenues entre 1979 et 1990. La dalle X pèse 7,6 tonnes (clichés : B. Hallégouët-1979; B. Fichaut-2005).

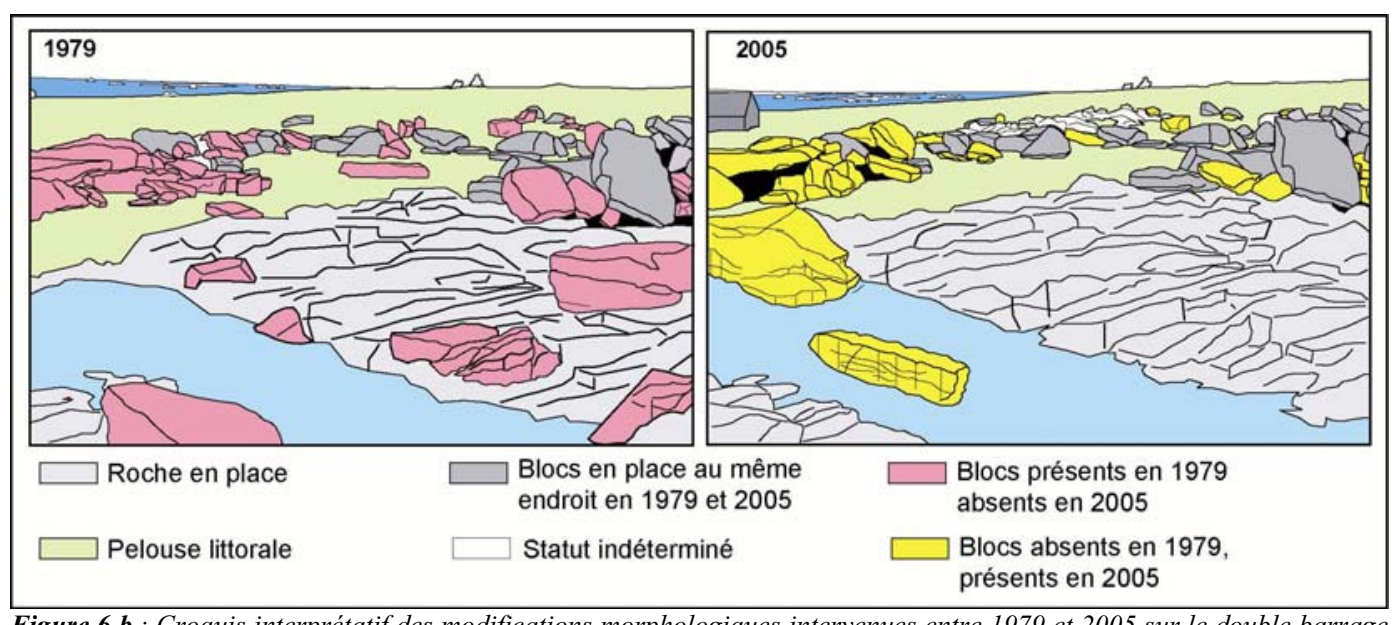

Figure 6 b : Croquis interprétatif des modifications morphologiques intervenues entre 1979 et 2005 sur le double barrage de blocs cyclopéens du centre de Banneg

\section{Conditions hydrodynamiques à l'origine de ces amas.}

Dans le but de caractériser les forçages responsables de ces amas, une étude des tempêtes de l'hiver 1989-1990 a été réalisée. En effet, les observations menées au printemps 1990 montrèrent qu'une grande partie des accumulations en place avait été remodelée. Plusieurs centaines de tonnes de dalles furent arrachées aux falaises et projetées à l'intérieur de l'île ${ }^{[6]}$.

L'étude des conditions hydrodynamiques s'appuie sur des données météomarines (vent et pression barométrique) acquises auprès de Météo France et des états de mer (houle et marégraphie) fournis respectivement par le CETMEF (Plouzané) et le SHOM (Brest). Trois épisodes tempétueux sont clairement identifiés entre le $1^{\text {er }}$ décembre 1989 et le 28 février 1990. La premier allant du 13 au 27 décembre 1989, est marqué par des hauteurs significatives de houles atteignant $10,5 \mathrm{~m}$ et $16 \mathrm{~m}\left(H_{\max }\right)$ et des surcotes proches de $90 \mathrm{~cm}$. Durant cet épisode, les pressions tombées à $970 \mathrm{hpa}$, ont généré des vents de $25 \mathrm{~m} / \mathrm{s}$. Lors du second évènement, du 23 janvier au 14 février 1990, on enregistre à deux reprises 
des $H_{\max }$ de houle de $18 \mathrm{~m}\left(H_{\text {sig }} 12 \mathrm{~m}\right)$ associées à des vents dépassant cinq fois 25 $\mathrm{m} / \mathrm{s}$. Les pressions un peu moins creusées (985 hpa) sont associées à des surcotes moins importantes de l'ordre de $50 \mathrm{~cm}$. Le dernier épisode survenu à la fin février 1990 montre des hauteurs de vagues plus faibles $\left(H_{\max } 14 \mathrm{~m}\right.$ et $\left.H_{\text {sig }} 8 \mathrm{~m}\right)$. Par contre les niveaux de pleine mer sont les plus importants de toute la période étudiée (marée observée atteignant 4 m IGN 69).

Notre intérêt s'est porté sur l'estimation des niveaux marins extrêmes ayant pu déplacer des blocs à des altitudes aussi importantes que celles relevées sur le terrain (entre 7 et $11 \mathrm{~m}$ IGN 69). La marée est le premier paramètre jouant sur l'élévation du niveau marin. Elle relève de paramètres astronomiques auxquels s'ajoutent les effets météorologiques (pression barométrique et vent) ${ }^{[7][8][9]}$. Aussi, la mesure de la marée observée illustre bien la combinaison de ces paramètres sur la déformation du plan d'eau. A la marée se surimpose l'action de la houle à la côte dont un des processus appelé runup est défini comme étant la somme de deux composantes: les variations du plan d'eau liées à l'agitation (setup : $\bar{\eta}_{\max }$ ) et l'action du jet de rive (wave uprush : $R$ ), soit $R^{T}=\bar{\eta}_{\text {max }}+R^{[10]}$. Le runup a été calculé à partir des équations de H. Mase ${ }^{[11]}$ :

$$
\begin{aligned}
\frac{R^{T} \text { max }}{H_{\text {sig }}} & =2,32 \xi_{o}^{0,77} \\
\frac{R^{T}{ }_{2 \%}}{H_{\text {sig }}} & =1,86 \xi_{o}^{0,71}
\end{aligned}
$$

où $L_{0}$ est la longueur d'onde en eau profonde, $R_{\text {max }}^{T}$ correspond au runup maximum et $R^{T}{ }_{2 \%}$ à celui dépassé par $2 \%$ des plus hauts jets de rive; $H_{\text {sig }}$ : hauteur significative au large (en mètres) ; constante $C=2,32$ pour $R_{\text {max }}^{T}$ et 1,86 pour $R_{2 \%}^{T} ; \xi_{o}$ : nombre d'Iribarren ${ }^{[12]}$ :

$$
\xi_{o}=\frac{\tan \beta}{\left(H_{\text {sig }} / L_{o}\right)^{1 / 2}}
$$

Les résultats obtenus sont présentés dans le tableau 2. Pour des pentes $(\tan \beta)$ de l'avant côte allant de 0,02 à 0,05 du nord au sud, le runup $R^{T}{ }_{2 \%}$ atteint

\begin{tabular}{|c|c|c|c|c|c|}
\hline$S(\tan \beta)$ & $H_{\text {sig }}(m)$ & $T_{\text {sig }}(s)$ & $\zeta_{o}$ & $R_{2 \%}^{T}$ & $R_{\max }^{T}$ \\
\hline 0,02 & \multirow[t]{2}{*}{$\max .12,2$} & \multirow[t]{2}{*}{$\max .18,5$} & $\max .0,25$ & $4,5 \mathrm{~m}$ & $5 \mathrm{~m}$ \\
\hline 0,05 & & & $\max .0,65$ & $8,7 \mathrm{~m}$ & $10 \mathrm{~m}$ \\
\hline
\end{tabular}
respectivement $4,5 \mathrm{~m}$ et $8,7 \mathrm{~m}$. Les valeurs maximum $R^{T}{ }_{\text {max }}$ sont respectivement égales à $5 \mathrm{~m}$ et $10 \mathrm{~m}$.

Comme le montre la figure 7, les niveaux extrêmes enregistrés durant les trois mois étudiés atteignent respectivement $8 \mathrm{~m}$ au nord et $12,5 \mathrm{~m}$ au centre de l'île. Ces valeurs représentent des ordres de grandeur, certainement inférieurs à la réalité, toutefois elles montrent que dans les deux secteurs les déferlantes ont atteint, voire dépassé le sommet de falaise. Les données sur la partie centrale montrent que la submersion a été plus importante et plus fréquente qu'au nord de 
l'île. Cela explique l'importance des accumulations dans ce secteur et confirme le rôle important de la morphologie de l'avant côte, et de l'orientation de cette dernière par rapport au fetch dominant, dans la dynamique morpho-sédimentaire.

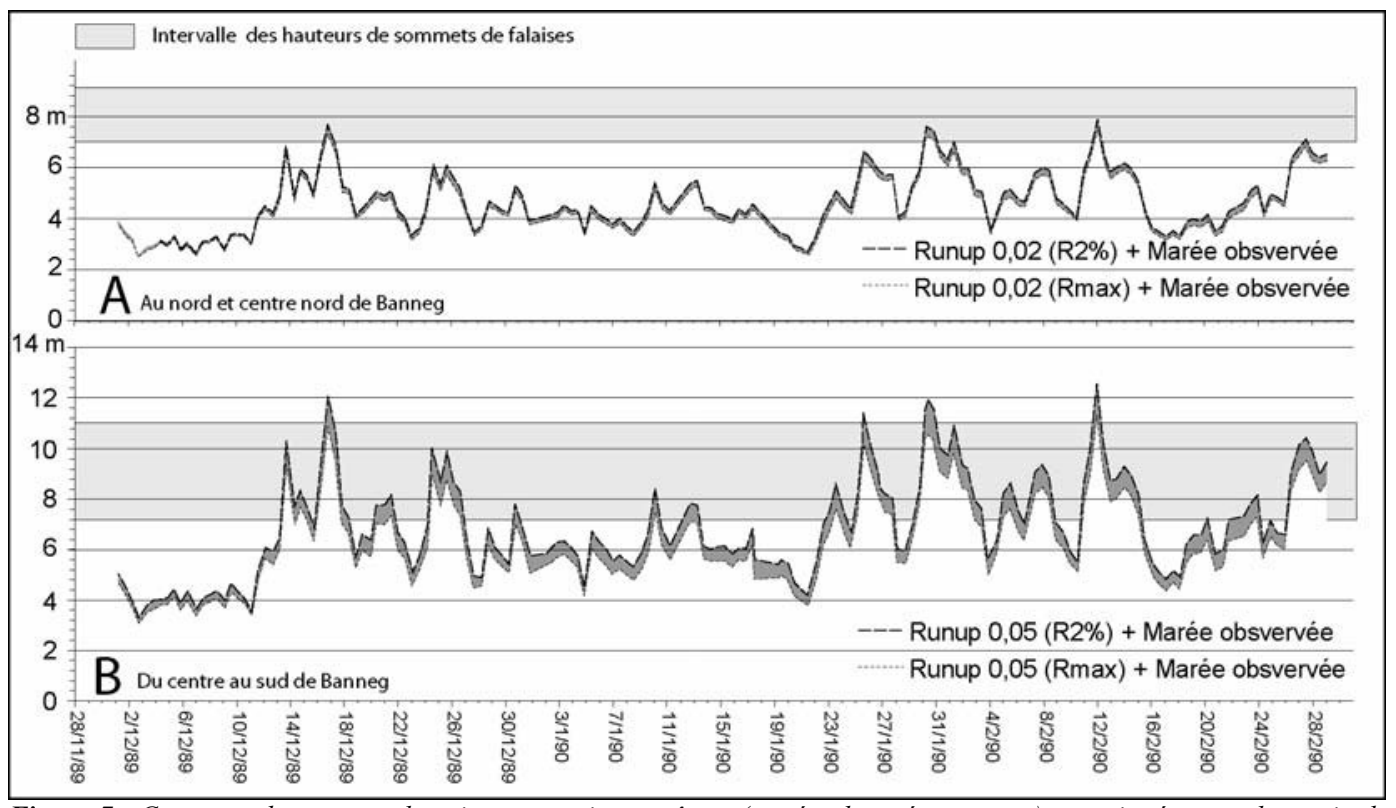

Figure 7: Correspondance entre les niveaux marins extrêmes (marée observée + runup) enregistrés entre les mois de décembre 1989 et février 1990 et la hauteur des falaises au nord de l'île (7A) et au centre - sud de l'île (7B).

\section{Conclusion}

Cette étude renouvelle l'état des connaissances en matière de dynamique morpho-sédimentaire dans l'archipel de Molène. A plus grande échelle, elle remet en cause l'idée largement répandue qui tendrait à attribuer la présence des amas de blocs cyclopéens à la seule action des vagues de tsunami. Ainsi, l'action morphogène des fortes tempêtes atlantiques est suffisamment efficace lorsque le contexte morphologique le permet. La suite de ce travail s'attachera à déterminer la récurrence de ces épisodes morphogènes en analysant sur le long terme les données météo-marines et des états de mer disponibles.

\section{Bibliographie}

1. Hallégouët B., (1984). Contribution à l'étude morphologique de l'archipel de

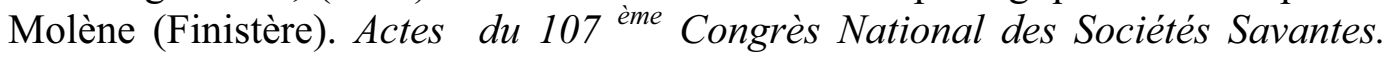
Brest 1982. Secteur de Géographie. Etudes géographiques sur la Bretagne et questions diverses. Paris, CTHS, 61 - 77

2. Guilcher A., (1959). L'archipel de Molène (Finistère). Etude morphologique. Revue de géographie Physique et de Géologie Dynamique, II, 2, 81-96.

3. Noormets R., Crook K., Felton E. (2004). Sedimentology of rocky shorelines : 3. Hydrodynamics of megaclast emplacement and transport on a shore platform, Oahu, Hawaii. Sedimentary Geology, 172, 41-65. 
4. Whelan F., Kelletat D., (2005). Boulder deposits on the southern spanish Atlantic coast : possible evidence for the 1755 AD Lisbon Tsunami ? Science of Tsunami Hazards, 23 (3), 25 - 38.

5. Jones B., Hunter I. G., (1992). Very large boulders on the coast of the Grand Cayman: the effects of giant waves on rocky coastlines. Journal of Coastal Research, 8 (4), 763-774.

6. Fichaut B., Hallégouët B., (1989). Banneg, une île dans la tempête, Penn ar Bed, 135, $36-43$.

7. Bouligand R., Pirazzoli P.A., (1999) - Les surcotes et les décotes marines à Brest, étude statistique et évolution, Oceanologica Acta, 22-2, 153-166.

8. Pirazzoli P.A., (2000) - Surges, atmospheric pressure and wind change and flooding probability on the Atlantic coast of France, Oceanologica Acta, 23-6, 643-661.

9. Betts N.L., Orford, J.D., White D., Graham C.J., (2004) - Storminess and surges in the South Western Approaches of the eastern North Atlantic: the synoptic climatology of recent extreme coastal storms, Marine Geology, 210, 227246.

10. Komar P.D., (1998) - Beach processes and sedimentation, $2^{\text {nd }}$ Edit., Printice Hall, 544p.

11. Mase H., (1989) - Random wave runup height on gentle slopes, J. Waterway, Port, Coastal, and Ocean Engineering, 115 (5), 649-661.

12. Battjes J.A. (1974) - Surf similarity, Proceeding of the 14th Coastal Engineering Conference, Amer. Soc. Civil Engrs, 466-479. 